\title{
Correction to: Semantic annotation of summarized sensor data stream for effective query processing
}

\author{
Shobharani Pacha ${ }^{1}$ - Suresh Ramalingam Murugan ${ }^{2}$. \\ R. Sethukarasi ${ }^{3}$
}

\section{Correction to: J Supercomput https://doi.org/10.1007/s11227-017-2183-7}

R. Sethukarasi was not listed among the authors. The original article has been corrected.

The original article can be found online at https://doi.org/10.1007/s11227-017-2183-7.

Shobharani Pacha

pachashobharani@gmail.com

1 RMD Engineering College, Chennai, India

2 Sri Lakshmi Ammal Engineering College, Chennai, India

3 R.M.K. Engineering College, Chennai, Tamil Nadu, India 